\title{
VALORES DE REFERÊNCIA DO DRIS PARA A SOJA, cv. CD 202, PARA A REGIÃO SUL DO MATO GROSSO DO SUL' ${ }^{1}$
}

\section{REFERENCES VALUES OF DRIS FOR SOYBEAN, cV.CD 202, IN THE SOUTH OF THE MATO GROSSO DO SUL STATE, BRAZIL}

\author{
Shizuo MAEDA ${ }^{2}$ \\ Pedro RONZELLI Júnior ${ }^{3}$ \\ Luiz Antônio Correa LUCCHESI ${ }^{4}$
}

\begin{abstract}
RESUMO
A adequação da fertilidade da maioria dos solos das áreas de cerrado do Mato Grosso do Sul para o cultivo da soja, requereu a aplicação intensa de calcários e fertilizantes. Os critérios mais utilizados para o manejo da fertilidade e a caracterização nutricional das culturas são as análises de solo e de folhas cujos resultados são interpretados com base em faixas de concentração. Porém, a eficiência de tais critérios são restringidas pela interpretação de resultados analíticos de forma isolada para cada nutriente $e$ sua não hierarquização quanto a limitações nutricionais, bem como possíveis efeitos de cultivar, idade de tecido e fatores edafoclimáticos locais. Para superar tais limitações, o Sistema Integrado de Diagnose e Recomendação (DRIS) surge como alternativa aos referidos critérios interpretativos. Embora sendo menos suscetível aos referidos problemas que interferem nos critérios mencionados, o DRIS também é influenciado pelos mesmos fatores, requerendo o desenvolvimento de valores de referência particularizados para situações regionais para minimizar tais deficiências. Com o objetivo de validar valores de referência do DRIS para soja cultivada no sul do Mato Grosso do Sul, amostras de folhas e grãos foram coletadas em lavouras comerciais na safra 2000/2001. Os valores de referência do DRIS foram validados por meio de sua aplicação em resultados de amostras foliares, que se apresentavam com sintoma de distúrbio nutricional. Os resultados obtidos mostram que: 1) os índices DRIS calculados com base nos valores de referência desenvolvidos no trabalho e os compilados da literatura são diferentes;2) a concordância entre os diagnósticos feitos pelos métodos da diagnose visual e do DRIS, com base em sintomas foliares caracterizados como provocados por deficiência de Mn, indicam a eficiência do DRIS e; 3) o DRIS é mais eficiente que o critério das faixas de concentração na interpretação de resultados analíticos de amostras de folhas.
\end{abstract}

Palavras-chave: Glycine max, nutrição mineral, DRIS, Mato Grosso do Sul/Brasil.

\begin{abstract}
In cerrado region of Mato Grosso do Sul state, Brazil, the intensive application of calcareous and fertilizer was necessary to soybean productions. Soil and leaf analysis have been the mostly used criteria for soil fertility management and nutritional status characterization. For that purpose, analytical results of soil and leaves sample was interpreted by means concentrations ranges criteria. However, it is well known that the resultant interpretation done in an isolated way for each nutrient by the critical concentration ranges criteria are difficult to be organized under a hierarchy of limitations and are affected by several factors such as variety, tissue age, climate and soil properties, points which constitute restrictions for the validity and quality of recommendations based on them. In order to overcome such limitations some authors have proposed an alternative interpretation method, the Diagnosis and Recommendation Integrated System (DRIS), considered less susceptible but yet influenced by some factors in its discriminatory ability and efficiency of diagnosing plant nutrition status, and thus requiring the development of reference values for its regional application. The aim of this work was to develop and to valid reference values for soybean crops cultivated in Mato Grosso do Sul state. For that purpose leaves and soybean grain were sampled in commercial areas, always from same sites. Regional reference values based on the leaf samples were validated by comparing DRIS interpretation with the visual diagnosis method. The efficacy and the ability to discriminate and to order nutritional problems were better for DRIS than for the other tested methods. This was demonstrated when data from a leaf sample presenting a classical Mn visual deficiency symptom led DRIS to rank Mn as the most limiting nutrient. On the other hand, the difference between outputs given by DRIS based on published values and DRIS based on regional values suggests that refinement of reference values may be beneficial when an improvement in DRIS accuracy is required..
\end{abstract}

Key-words: Glycine max, mineral nutrition, DRIS, Mato Grosso do Sul/Brazil.

${ }^{1}$ Parte do trabalho de tese de Doutorado do primeiro autor.

${ }^{2}$ Eng $^{\circ}$ Agroํ․ Doutor, CREA 14.915/D, PR, Pesquisador da EMBRAPA Florestas. Estrada da Ribeira, km 111, CP 319, Colombo (PR), CEP 83411-000. E-mail: maeda@cnpf.embrapa.br

${ }^{3}$ Eng $^{\circ}$ Agr ${ }^{\circ}$, Doutor, CREA 37.209/D, 6ª Região, Visto 5.867-PR, Professor do Departamento de Fitotecnia e Fitossanitarismo, Universidade Federal do Paraná.

${ }^{4} E_{n g}{ }^{\circ}$ Agr ${ }^{\circ}$, PhD, CREA 10457/D, PR, Professor Adjunto do Departamento de Solos e Engenharia Agrícola, Universidade Federal do Paraná. 


\section{INTRODUÇÃO}

No Mato Grosso do Sul, a maioria dos solos utilizados para o cultivo da soja era, originalmente, coberta pela vegetação de cerrado e caracterizavamse pela baixa fertilidade natural, o que exigiu a sua adequação, com a aplicação maciça de calcários e fertilizantes, para a viabilização econômica de tal atividade. Ao longo dos anos, o manejo da fertilidade do solo, para o cultivo da soja têm-se baseado em análises de solo e de folhas, cujos resultados analíticos são interpretados a partir do critério das faixas de concentração. O diagnóstico nutricional realizado por este método, baseia-se na comparação da concentração de cada nutriente analisado na amostra em diagnose com as respectivas faixas de concentração crítica. Em geral, na interpretação de resultados analíticos de amostras foliares, a concentração de cada nutriente é considerada de forma independente, não sendo observada as proporções entre as concentrações dos nutrientes para a diagnose nutricional, o que se constitui numa limitação do método. Além disso, estando dois ou mais nutrientes abaixo de suas faixas de concentração críticas, o método não permite hierarquizar essas limitações. A diagnose nutricional por esse critério também é limitada pela idade do tecido amostrado e por variações nas concentrações de nutrientes entre cultivares.

O Sistema Integrado de Diagnose e Recomendação - DRIS, desenvolvido por Beaufils (1973), apresenta características que permitem minimizar as deficiências apresentadas pelo método das faixas de concentração e constitui-se numa alternativa para os referidos critérios interpretativos. O DRIS é um sistema de cálculo no qual razões entre concentrações de nutrientes em uma amostra são comparados matematicamente com valores "ótimos" (valores de referência ou normas) das mesmas razões obtidas a partir de uma população de alta produtividade (população de referência) a qual é assumida como nutricionalmente equilibrada. A aplicação do método possibilita a obtenção de índices de diagnose utilizados no ordenamento dos desequilíbrios nutricionais. Quando obtidas em condições de ampla variabilidade ambiental, os valores de referência do DRIS eram considerados como de aplicação universal. Porém, tais valores têm mostrado efeito de fatores edafoclimáticos regionais, o que têm resultado em diagnósticos errôneos quando os mesmos são obtidos em condições distintas daquela da amostra sob diagnose. Tal fato, indica a necessidade de se obter valores de referência particularizados para o incremento da eficiência do DRIS, conforme observado por Beaufils (1973). Embora apresente as deficiências indicadas anteriormente, estudos com o DRIS, realizados por Leandro (1998) e Sumner (1979), têm indicado boas perspectivas para a diagnose nutricional.

O objetivo do presente trabalho foi o de validar valores de referência do DRIS para a soja cultivada no sul do Mato Grosso do Sul.

\section{METODOLOGIA}

Para o desenvolvimento do trabalho, amostras de folhas e de grãos foram coletadas na safra 2000/ 2001, em lavouras comerciais na região sul do Mato Grosso do Sul, considerada neste trabalho como a região ao sul do paralelo $21^{\circ} \mathrm{S}$. Oitenta e sete amostras foram coletadas em 46 propriedades, selecionadas com base em dois critérios: manejo adequado da lavoura e semeadura da cultivar CD 202. A amostragem de folhas foi realizada no estádio fenológico R1 (Costa e Marquezan, 1982), coletandose a última folha amadurecida com pecíolo (terceira folha, do ápice para a base da planta, na haste principal) de acordo com o recomendado por Borkert et al. (1994) e Bataglia e Dechen (1986). Nas amostras foliares foram determinados os teores totais de $\mathrm{N}, \mathrm{P}$, $\mathrm{K}, \mathrm{Ca}, \mathrm{Mg}, \mathrm{S}, \mathrm{B}, \mathrm{Cu}, \mathrm{Fe}, \mathrm{Mn}$ e $\mathrm{Zn}$, conforme metodologia descrita em Malavolta et al. (1989). Para a determinação da produtividade de grãos foram colhidas plantas em oito segmentos de um metro nas fileiras de cada parcela. Esse procedimento foi realizado na maturação de colheita o qual corresponde ao estádio R8 na escala de Costa e Marquezan (1982). As plantas colhidas foram secas e trilhadas e em seguida as amostras de grãos foram avaliadas para produtividade, sendo os valores expressos $\mathrm{kg} \cdot \mathrm{ha}^{-1}$, a $13 \%$ de umidade.

O DRIS foi desenvolvido conforme os passos sugeridos por Schutz e Villiers (1987), quais sejam: a) formação do banco de dados; b) seleção da população de referência e obtenção dos valores de referência; c) cálculo dos índices de diagnose; e d) avaliação dos valores de referência estabelecidos.

\section{Formação do banco de dados}

O banco de dados utilizado para o desenvolvimento dos valores de referência do DRIS, para a região sul do Mato Grosso do Sul, foi composto por 87 amostras. As informações utilizadas para a formação da base de dados e para o desenvolvimento do DRIS foram os teores totais de nutrientes nas folhas e produtividade de grãos.

\section{Seleção da população de referência}

O banco de dados foi dividido em duas subpopulações, considerando-se a produtividade de $3.500 \mathrm{~kg} \cdot$ ha $^{-1}$ como o limite entre a subpopulação A, de alta produtividade $\left(\geq 3.500 \mathrm{~kg} \cdot \mathrm{ha}^{-1}\right)$ a qual corresponde à população de referência do DRIS e a subpopulação $B$, de baixa produtividade $\left(<3.500 \mathrm{~kg} \cdot \mathrm{ha}^{-1}\right)$.

\section{Obtenção dos valores de referência}

Em cada sub-população os nutrientes foram expressos em todas as relações possíveis na forma de quociente e, em seguida, foram calculadas as médias $\left(X_{A}\right.$ e $\left.X_{B}\right)$, os desvios-padrão $\left(s_{A}\right.$ e $\left.s_{B}\right)$, os 
MAEDA, S.; RONZELLI Jr., P. Valores de referência do dris para...

coeficientes de variação $(\mathrm{CV})$ e as variâncias $\left(\mathrm{s}_{\mathrm{A}}{ }^{2} \mathrm{e}\right.$ $\mathrm{S}_{\mathrm{B}}{ }^{2}$ ) da concentração de cada nutriente e dos valores dos quocientes das relações entre estes, de acordo com Walworth e Summer (1987) e Beaufils (1973). Por meio da comparação da razão entre as variâncias das relações nutricionais das duas subpopulações $\left(\mathrm{s}_{\mathrm{B}}{ }^{2} / \mathrm{s}_{\mathrm{A}}{ }^{2}\right)$, para cada relação nutricional, na forma direta e inversa (exemplo N/P e P/N), foram selecionadas aquelas relações com maior razão entre as variâncias, conforme Walworth e Sumner (1987). As médias dos quocientes das relações entre as concentrações dos nutrientes selecionados e os seus respectivos desvios padrão e coeficientes de variação, correspondentes à subpopulação de alta produtividade $(A)$, representam os valores ou normas de referência do DRIS.

Os valores de referência, desenvolvidos nesse trabalho, foram comparados com aqueles mencionados por Leandro (1998), obtidos para a região de Rio Verde, GO. A comparação entre os valores de referência foi feita pelo teste $t$, enquanto que a verificação da normalidade na distribuição dos dados foi feita por meio da prova de aderência à distribuição normal de Kolmogorov-Smirnov.

\section{Cálculo dos índices de diagnose do DRIS}

As amostras com produtividades inferiores a $3.500 \mathrm{~kg} \cdot \mathrm{ha}^{-1}$ foram submetidas à diagnose pelo DRIS, com base nos valores de referência deste trabalho e de Leandro (1998). O cálculo do índice de diagnose do DRIS, para cada nutriente, foi feito por intermédio da fórmula geral proposta por Beaufils (1973), conforme a Equação 1:

Equação (1)

ÍndiceX $=\left[\frac{f(X / Y 1)+\ldots+f(X / Y m)-f(Z 1 / X)-\ldots-f(Z n / X)}{m+n}\right]$

sendo:

$X=$ nutriente genérico sob avaliação;

$\mathrm{Y} 1, \mathrm{Y} 2$ e $\mathrm{Yn}=$ nutrientes genéricos que aparecem no denominador das relações com o nutriente genérico $X$;

$\mathrm{Z1}, \mathrm{Z2}$ e $\mathrm{Zn}$ = nutrientes genéricos que aparecem no numerador das relações com o nutriente genérico $X$;

$\mathrm{m}$ = número de funções em que o nutriente em processamento se encontra no numerador da relação;

$\mathrm{n}$ = número de funções em que o nutriente em processamento se encontra no denominador da relação;

$f(X / Y 1)=$ função da relação entre os nutrientes genéricos $\mathrm{X}$ e $\mathrm{Y} 1 \mathrm{e}$,

$f(Z 1 / X)=$ função da relação entre os nutrientes genéricos $Z 1$ e $X$.

Por outro lado, o cálculo das funções foi feito conforme Elwali e Gascho (1984):

Equação (2)

$$
f(X / Y)=\left[\left(\frac{X / Y}{X / y}\right)-1\right]\left(\frac{100 k}{C V}\right) \text { se } X / Y>x / y+s_{x / y}
$$

Equação (3)

$$
f(X / Y)=\left[1-\left(\frac{X / y}{X / Y}\right)\right]\left(\frac{100 k}{C V}\right) \text { se } X / Y<x / y-s_{x / y}
$$

Equação (4)

$$
f(X / Y)=0 \text { se } x / y-s_{x / y} \leq X / Y \leq x / y+s_{x / y}
$$

sendo:

$\mathrm{X} / \mathrm{Y}=$ valor do quociente das concentrações entre dois nutrientes genéricos $\mathrm{X}$ e $\mathrm{Y}$ na planta sob avaliação;

$x / y=$ valor de referência entre dois nutrientes genéricos $\mathrm{X}$ e $\mathrm{Y}$;

CV = coeficiente de variação da relação entre dois nutrientes genéricos $X$ e $Y$ da população de referência;

$k=$ coeficiente de sensibilidade, de valor arbitrário, utilizado nesta equação de acordo com Beaufils (1973), para que os valores dos índices de diagnose apresentem magnitudes convenientes, sendo o valor adotado neste trabalho igual a 10, e

$\mathrm{S}_{x / y}=$ desvio-padrão da população de referência - subpopulação A.

Os sinais das funções inseridas na fórmula de cálculo dos índices de diagnose foram atribuídos considerando-se a posição do nutriente na relação. No numerador o sinal é positivo e no denominador é negativo (BEAUFILS, 1973). 


\section{Avaliação dos valores de referência desenvolvidos e comparação entre o DRIS e o critério das faixas de concentração}

A avaliação dos valores de referência foi baseada na verificação da capacidade dos mesmos em indicar, por meio dos índices de diagnose do DRIS, qual o nutriente estaria em nível capaz de induzir o desenvolvimento dos sintomas de deficiência observados. O procedimento de avaliação dos valores de referência do DRIS, conforme mencionado por Bataglia e Santos (1990), baseou-se na comparação entre o resultado da diagnose visual dos sintomas apresentados pelas folhas e a diagnose proporcionada pelos índices do DRIS. Ocorrendo a concordância entre os diagnósticos os valores de referência do DRIS foram considerados válidos.

Para a realização da avaliação proposta foram coletadas amostras de folhas que apresentavam sintomas de deficiência nutricional, em lavoura de produtor da mesma cultivar amostrada para o desenvolvimento dos valores de referência, procedimento também adotado por Leandro (1998). As folhas coletadas apresentavam um amarelecimento internerval, conforme mostrado na Fig. 1, cuja descrição encontra-se citada em Borkert et al. (1994), que caracterizam o sintoma como o provocado pela deficiência de Mn. As amostras de folhas, coletadas juntamente com as amostras de solo do local de ocorrência dos sintomas descritos, foram analisadas quimicamente, conforme procedimentos descritos por Malavolta et al. (1989) e Embrapa Solos (1999), respectivamente, para as amostras de folhas e solo. Os resultados analíticos das amostras de folhas foram interpretados pelos critérios das faixas de concentração e DRIS, enquanto que, as de solo foram interpretadas conforme Embrapa Agropecuária Oeste (2000).

\section{RESULTADOS E DISCUSSÃO}

São apresentados na Tabela 1, as médias dos teores foliares dos nutrientes correspondentes à população de referência deste trabalho bem como os apresentados por Leandro (1998), obtidos na região de Rio Verde, GO.

Dos nutrientes que apresentaram diferenças significativas nos teores médios entre a população de referência desse trabalho e aquelas obtidas por Leandro (1998) podem se destacar o $\mathrm{K} \mathrm{e} \mathrm{o} \mathrm{Fe}$. Os elevados valores dos teores médios de $\mathrm{K}(31,1 \mathrm{~g} . \mathrm{kg}$ $\left.{ }^{1}\right)$ e $\mathrm{Fe}\left(268,7 \mathrm{mg} \cdot \mathrm{kg}^{-1}\right)$, obtidos por Leandro (1998), são, segundo o autor, resultados de elevadas doses de $\mathrm{K}$ aplicadas nas glebas mais produtivas e de elevados teores de Fe encontrados nos solos da região estudada, em função da riqueza do material de origem de seus solos. Os altos teores foliares de $\mathrm{K} \mathrm{e} \mathrm{Fe}$, observados por Leandro (1998), podem superestimar os VR das relações nutricionais em que $\mathrm{K}$ ou $\mathrm{Fe}$ estiverem no numerador na relação nutricional $e$ subestimar quando os nutrientes estiverem no denominador da relação, resultando em erros de interpretação pelo DRIS.

TABELA 1 - Teores foliares médios de nutrientes da população de referência, obtidos em amostras coletadas no sul do Mato Grosso do Sul comparados com os desenvolvidos por Leandro (1998).

\begin{tabular}{|c|c|c|c|c|c|}
\hline \multirow[t]{2}{*}{ Variável } & \multicolumn{2}{|c|}{ Média } & \multirow[t]{2}{*}{ Variável } & \multicolumn{2}{|c|}{ Média } \\
\hline & $A^{(1)}$ & $\mathrm{B}^{(2 ; 3)}$ & & $A^{(1)}$ & $\mathrm{B}^{(2 ; 3)}$ \\
\hline $\mathrm{N}\left(\mathrm{g} \cdot \mathrm{kg}^{-1}\right)$ & 37,0 & $39,3^{*}$ & $\mathrm{~B}\left(\mathrm{mg} \cdot \mathrm{kg}^{-1}\right)$ & 39,4 & \\
\hline$P\left(g \cdot \mathrm{kg}^{-1}\right)$ & 2,7 & 2,9 & $\mathrm{Cu}\left(\mathrm{mg} \cdot \mathrm{kg}^{-1}\right)$ & 9,8 & 10,27 \\
\hline $\mathrm{K}\left(\mathrm{g} \cdot \mathrm{kg}^{-1}\right)$ & 22,4 & 31,1 * & $\mathrm{Fe}\left(\mathrm{mg} \cdot \mathrm{kg}^{-1}\right)$ & 82,2 & 268,74 * \\
\hline $\mathrm{Ca}\left(\mathrm{g} \cdot \mathrm{kg}^{-1}\right)$ & 11,5 & $8,1^{*}$ & $\mathrm{Mn}\left(\mathrm{mg} \cdot \mathrm{kg}^{-1}\right)$ & 62,6 & 39,15 * \\
\hline $\operatorname{Mg}\left(g \cdot \mathrm{kg}^{-1}\right)$ & 3,8 & $4,7^{*}$ & $\mathrm{Zn}\left(\mathrm{mg} \cdot \mathrm{kg}^{-1}\right)$ & 62,8 & 38,89 * \\
\hline$S\left(g \cdot \mathrm{kg}^{-1}\right)$ & 2,5 & 1,8 * & 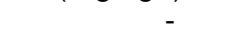 & - & - \\
\hline
\end{tabular}

(1) valores obtidos neste trabalho;

(2) teste t para médias sendo: * = significativo a 5 \% e ausência de asterisco = não significativo;

(3) Leandro (1998).

Na Tabela 2, são apresentados os valores foliares de referência - VR (média e coeficiente de variação) para o DRIS obtidos neste trabalho. Os mencionados VR foram comparados com os obtidos por Leandro (1998) (Tabela 3). A comparação, pelo teste t, entre os VR obtidos com os de Leandro (1998), mostra que, das 45 relações nutricionais que foram coincidentes, $39(86,6 \%)$ delas apresentaram diferenças estatísticas a $5 \%$ de probabilidade.

Vários fatores podem influenciar a concentração de nutrientes na planta e portanto os VR do DRIS, a saber: idade da planta; idade e parte da planta amostrada; clima (temperatura, quantidade e distribuição das chuvas, duração do dia e da noite e horário de coleta da amostra); fatores edáficos; incidência de moléstias e pragas e práticas culturais (BATAGLIA et al., 1996 e KEOGH et al., 1972). Além dos fatores mencionados anteriormente, a cultivar amostrada pode ter efeito sobre a concentração de nutrientes na folha, conforme Keogh et al. (1972), o que pode provocar alterações dos VR do DRIS. Leandro (1998) coletou amostras de folhas em lavouras da cultivar Cristalina, enquanto neste trabalho a coleta foi realizada em lavouras da cultivar CD 202. 
MAEDA, S.; RONZELLI Jr., P. Valores de referência do dris para...

TABELA2 - Valores foliares de referência (média e coeficiente de variação - CV) do DRIS desenvolvidos neste trabalho com base em amostras coletadas na região sul do Mato Grosso do Sul.

\begin{tabular}{|c|c|c|c|c|c|c|c|c|}
\hline Variável & Média & CV (\%) & Variável & Média & CV (\%) & Variável & Média & CV (\%) \\
\hline $\mathrm{N} / \mathrm{P}$ & 13,9 & 21,7 & $\mathrm{~K} / \mathrm{Ca}$ & 2,05 & 32,9 & $\mathrm{Mn} / \mathrm{Mg}$ & 16,97 & 44,2 \\
\hline $\mathrm{K} / \mathrm{N}$ & 0,62 & 22,9 & $\mathrm{~K} / \mathrm{Mg}$ & 6,17 & 30,1 & $\mathrm{Mg} / \mathrm{Zn}$ & 0,08 & 51,0 \\
\hline $\mathrm{N} / \mathrm{Ca}$ & 3,36 & 25,2 & $\mathrm{~K} / \mathrm{S}$ & 9,29 & 27,0 & $\mathrm{~B} / \mathrm{S}$ & 16,34 & 24,6 \\
\hline $\mathrm{N} / \mathrm{Mg}$ & 9,98 & 20,5 & $B / K$ & 1,83 & 27,5 & $\mathrm{Cu} / \mathrm{S}$ & 3,89 & 31,1 \\
\hline $\mathrm{N} / \mathrm{S}$ & 15,22 & 20,1 & $\mathrm{Cu} / \mathrm{K}$ & 0,45 & 37,4 & $\mathrm{Fe} / \mathrm{S}$ & 34,27 & 45,9 \\
\hline $\mathrm{B} / \mathrm{N}$ & 1,08 & 18,4 & $\mathrm{Fe} / \mathrm{K}$ & 3,82 & 45,3 & $\mathrm{Mn} / \mathrm{S}$ & 25,90 & 45,3 \\
\hline $\mathrm{Cu} / \mathrm{N}$ & 0,27 & 39,3 & $\mathrm{Mn} / \mathrm{K}$ & 2,82 & 39,5 & $S / Z n$ & 0,05 & 43,3 \\
\hline $\mathrm{Fe} / \mathrm{N}$ & 2,23 & 39,6 & $\mathrm{~K} / \mathrm{Zn}$ & 0,43 & 44,6 & $\mathrm{~B} / \mathrm{Cu}$ & 4,78 & 50,0 \\
\hline $\mathrm{Mn} / \mathrm{N}$ & 1,71 & 39,6 & $\mathrm{Mg} / \mathrm{Ca}$ & 0,34 & 23,9 & $\mathrm{~B} / \mathrm{Fe}$ & 0,56 & 43,2 \\
\hline $\mathrm{N} / \mathrm{Zn}$ & 0,72 & 44,2 & $\mathrm{Ca} / \mathrm{S}$ & 4,75 & 29,7 & $\mathrm{Mn} / \mathrm{B}$ & 1,61 & 40,2 \\
\hline $\mathrm{K} / \mathrm{P}$ & 8,51 & 31,1 & $\mathrm{~B} / \mathrm{Ca}$ & 3,61 & 31,4 & $\mathrm{~B} / \mathrm{Zn}$ & 0,76 & 43,2 \\
\hline $\mathrm{Ca} / \mathrm{P}$ & 4,31 & 26,6 & $\mathrm{Cu} / \mathrm{Ca}$ & 0,88 & 41,9 & $\mathrm{Fe} / \mathrm{Cu}$ & 10,43 & 68,5 \\
\hline $\mathrm{Mg} / \mathrm{P}$ & 1,45 & 30,9 & $\mathrm{Fe} / \mathrm{Ca}$ & 7,37 & 46,6 & $\mathrm{Mn} / \mathrm{Cu}$ & 7,51 & 69,6 \\
\hline S/P & 0,95 & 49,4 & $\mathrm{Ca} / \mathrm{Mn}$ & 0,21 & 45,4 & $\mathrm{Cu} / \mathrm{Zn}$ & 0,19 & 54,5 \\
\hline $\mathrm{B} / \mathrm{P}$ & 14,86 & 25,4 & $\mathrm{Ca} / \mathrm{Zn}$ & 0,22 & 37,4 & $\mathrm{Mn} / \mathrm{Fe}$ & 0,88 & 57,9 \\
\hline $\mathrm{Cu} / \mathrm{P}$ & 3,74 & 49,4 & $\mathrm{Mg} / \mathrm{S}$ & 1,57 & 24,4 & $\mathrm{Fe} / \mathrm{Zn}$ & 1,48 & 45,4 \\
\hline $\mathrm{Fe} / \mathrm{P}$ & 30,29 & 37,9 & $\mathrm{~B} / \mathrm{Mg}$ & 10,75 & 26,8 & $\mathrm{Mn} / \mathrm{Zn}$ & 1,14 & 50,0 \\
\hline $\mathrm{Mn} / \mathrm{P}$ & 23,90 & 48,8 & $\mathrm{Mg} / \mathrm{Cu}$ & 0,46 & 46,7 & - & & - \\
\hline $\mathrm{P} / \mathrm{Zn}$ & 0,05 & 42,1 & $\mathrm{Fe} / \mathrm{Mg}$ & 22,42 & 44,3 & - & - & - \\
\hline
\end{tabular}

TABELA3 - Comparação entre os valores de referência do DRIS deste trabalho com as de Leandro (1998).

\begin{tabular}{lccc}
\hline Fontes & Número de relações & $\begin{array}{c}\text { Número de relações } \\
\text { coincidentes }\end{array}$ & $\begin{array}{c}\text { Percentagem de relações } \\
\text { diferentes }\end{array}{ }^{(1)}$ \\
\hline Deste trabalho & 55 & - & - \\
Leandro (1998) & 55 & 45 & 86,6 \\
\hline
\end{tabular}

(1) Teste t a $5 \%$ de propabilidade.

A produtividade de grãos como ponto de divisão da população de amostras também pode constituir-se num fator de diferenciação dos VR do DRIS. Os VR de Leandro (1998) foram para glebas com produtividade igual ou superior a $3.000 \mathrm{~kg} \cdot \mathrm{ha}^{-1}$. Nesse trabalho foram de amostras com produtividade superior a $3.500 \mathrm{~kg} \cdot \mathrm{ha}^{-1}$.

Mais importante que as diferenças observadas entre os VR estabelecidos sob diferentes condições edáficas é a interpretação dos índices de diagnose do DRIS de cada nutriente sob análise. Conforme pode ser observado na Tabela 4, o diagnóstico realizado em amostras de lavouras com produtividades inferiores a $3.500 \mathrm{~kg} \cdot$ ha $^{-1}$ coletadas na região sul do Mato Grosso do Sul, feitos com base nos VR obtidos por Leandro (1998) em trabalho realizado na região de Rio Verde, GO, e os desse trabalho mostram resultados distintos.

TABELA 4 - Índices DRIS calculados com base nos valores de referências-VR desse trabalho e com base nos VR obtidos por Leandro (1998) em amostras coletadas em lavouras de soja, cultivar CD 202, com produtividade de grãos até $3.500 \mathrm{~kg}$ ha $^{-1}$ no sul do Mato Grosso do Sul.

\begin{tabular}{|c|c|c|c|c|c|c|c|c|c|c|}
\hline Amostra & $\mathrm{N}$ & $\mathrm{P}$ & $\mathrm{K}$ & $\mathrm{Ca}$ & $\mathrm{Mg}$ & $\mathrm{S}$ & $\mathrm{Cu}$ & $\mathrm{Fe}$ & $\mathrm{Mn}$ & $\mathrm{Zn}$ \\
\hline & \multicolumn{10}{|c|}{ Calculados com base nos VR obtidos neste trabalho } \\
\hline 2 & -40 & -23 & -3 & 20 & 4 & -66 & -38 & -10 & 156 & 1 \\
\hline 25 & 52 & 39 & -5 & 40 & 46 & 58 & 15 & 16 & -106 & -155 \\
\hline 32 & 21 & -6 & -15 & -6 & 47 & 3 & -36 & -27 & 25 & -6 \\
\hline 90 & -24 & -109 & 39 & 1 & 39 & -24 & 28 & -39 & 103 & -13 \\
\hline 67 & -14 & 46 & -2 & -26 & -21 & -3 & -117 & 100 & 68 & -31 \\
\hline \multicolumn{11}{|c|}{ Calculados com base nos VR obtidos por Leandro (1998) } \\
\hline 2 & 137 & 113 & 86 & 200 & 66 & 140 & -278 & -382 & 1 & -83 \\
\hline 25 & 287 & 202 & 90 & 259 & 123 & 410 & -188 & -461 & -279 & -444 \\
\hline 32 & 247 & 151 & 96 & 209 & 119 & 268 & - 303 & -581 & -53 & -154 \\
\hline 90 & 165 & 63 & 129 & 202 & 108 & 222 & -120 & -602 & -13 & -155 \\
\hline 67 & 169 & 157 & 83 & 134 & 29 & 200 & -400 & -176 & -46 & -168 \\
\hline
\end{tabular}


Esses resultados demonstram que os índices de diagnose do DRIS, calculados a partir de VR obtidos em condições edafoclimáticas, de manejo e outros fatores diferentes daqueles de uma determinada amostra sob diagnose, podem apresentar valores diferentes para os índices de diagnose e, portanto, o diagnóstico poderá ser errôneo, reforçando a necessidade de se obter $V R$ regionalizados e particularizados para os fatores que o influenciam e por conseqüência a diagnose (BEAUFILS, 1973 e
LEANDRO, 1998).

São apresentados na Tabela 5, os índices de diagnose do DRIS dos nutrientes analisados na amostra de folhas e na Tabela 6 , os resultados analíticos referentes às amostras de solo e de folhas coletadas no local de aparecimento dos sintomas de distúrbio nutricional e os correspondentes diagnósticos realizados com base no critério das faixas de concentração. As folhas com distúrbios nutricionais são apresentadas na Figura 1.

TABELA 5 - Índices DRIS e ordem de deficiência dos nutrientes analisados em amostra de folhas de soja coletada em área cultivada com a cultivar CD 202, no sul do Mato Grosso do Sul, Dourados, MS, 2000/2001.

\begin{tabular}{|c|c|c|c|c|c|c|c|c|c|c|}
\hline \multicolumn{11}{|c|}{ Nutriente } \\
\hline $\mathrm{N}$ & $\mathrm{P}$ & $\mathrm{K}$ & $\mathrm{Ca}$ & $\mathrm{Mg}$ & $\mathrm{S}$ & $B$ & $\mathrm{Cu}$ & $\mathrm{Fe}$ & $\mathrm{Mn}$ & $\mathrm{Zn}$ \\
\hline \multicolumn{11}{|c|}{ Índice DRIS } \\
\hline 14 & 12 & -18 & 9 & 13 & 14 & -10 & -19 & 54 & -46 & -22 \\
\hline \multicolumn{11}{|c|}{ Ordem de limitação } \\
\hline & & & & & $>\mathrm{C}$ & & & & & \\
\hline
\end{tabular}

Avaliação do valores de referência e comparação do DRIS com o critérios das faixas de concentração baseada na diagnose visual, conforme descrição de sintomas feita por Borkert et al. (1994) caracterizaram-se os sintomas de distúrbios foliares como aqueles provocados pela deficiência de $\mathrm{Mn}$ (Figura 1). Os índices de diagnose do DRIS, estimados com base nos VR obtidos nesse trabalho, permitiram hierarquizar a ordem de limitação nutricional. Com base nessa hierarquização observouse que o $\mathrm{Mn}$ foi o nutriente mais limitante, o que concorda com a diagnose visual e valida os VR obtidos no trabalho. Seguiram-se em ordem de importância $\mathrm{Zn}, \mathrm{Cu}, \mathrm{K}$ e B.

TABELA6 - Resultados de análises de solo e de folhas de soja, de amostra coletada em área cultivada com a cultivar CD 202, no sul do Mato Grosso do Sul, Dourados, MS, 2000/2001.

\begin{tabular}{lcccccccccccc} 
Análise & \multicolumn{1}{c}{ Nutriente $^{(1)}$} & \multicolumn{1}{c}{ Diagnose $^{(2)}$} \\
\cline { 2 - 12 } & $\mathrm{N}$ & $\mathrm{P}$ & $\mathrm{K}$ & $\mathrm{Ca}$ & $\mathrm{Mg}$ & $\mathrm{S}$ & $\mathrm{B}$ & $\mathrm{Cu}$ & $\mathrm{Fe}$ & $\mathrm{Mn}$ & Zn & \\
\hline Solo & - & 47 & 0,2 & 3,0 & 1,1 & - & - & 1,3 & - & 14,2 & 3,3 & sem deficiência \\
Folha & 46,2 & 3,4 & 16 & 13,7 & 35 & 3,1 & 35 & 5,6 & 23,4 & 23,3 & 31,4 & $\mathrm{~K}$ e Cu - baixos \\
\hline
\end{tabular}

(1) unidades: $\mathrm{K}, \mathrm{Ca}$ e Mg no solo - $\mathrm{cmol}_{\mathrm{c}} \cdot \mathrm{dm}^{-3}$ e P, Cu, Mn e Zn no solo - mg.dm ${ }^{-3}$, N, P, K, Ca, Mg e S na folha em g.kg-1 e B, Cu, Fe, Mn e Zn na folha em mg.kg ${ }^{-1}$.

${ }^{(2)}$ de acordo com Embrapa Agropecuária Oeste (2000) para teores no solo e Peck (1979), para teores foliares.

A interpretação dos resultados da análise do solo da parcela amostrada, com base no método das faixas de concentração, proposta para o sul do Mato Grosso do Sul, não identificou teores abaixo dos níveis considerados suficientes para quaisquer dos nutrientes analisados, assim não se esperava a ocorrência de deficiência nutricional nas plantas cultivadas na área amostrada.

Por outro lado, a diagnose com base nos teores foliares considerando as faixas de concentração propostas por Peck (1979) e utilizadas no sul do Mato Grosso do Sul (EMBRAPAAGROPECUÁRIA OESTE, 2000) indicou que os teores de $\mathrm{K}$ e $\mathrm{Cu}$ enquadraram-se na classe de teores baixos. Nessa classe de teor não é esperado o aparecimento de sintomas de deficiência, o que caracterizaria uma condição nutricional de fome oculta ou de deficiência latente (TISDALE et al., 1993), ou seja, os teores de $\mathrm{K}$ e $\mathrm{Cu}$ estariam acima da faixa de aparecimento de sintomas de deficiência nutricional, porém, ainda abaixo do necessário para a ótima produtividade, o que contrasta com o aparecimento de sintomas de deficiência nutricional, conforme mostrado na Figura 1.

Pelo menos duas hipóteses podem ser consideradas para a diferença entre as diagnoses feitas com base nos resultados analíticos das amostras de solo e de folhas interpretadas pelas faixas de concentração: a) as faixas de suficiência definidas para os teores de $\mathrm{K}$ e de $\mathrm{Cu}$ no solo e na folha citadas em Embrapa Agropecuária Oeste (2000) não são adequadas para o solo em questão, e b) condições ambientais e genéticas limitaram a absorção dos nutrientes pelas plantas. 
MAEDA, S.; RONZELLI Jr., P. Valores de referência do dris para...

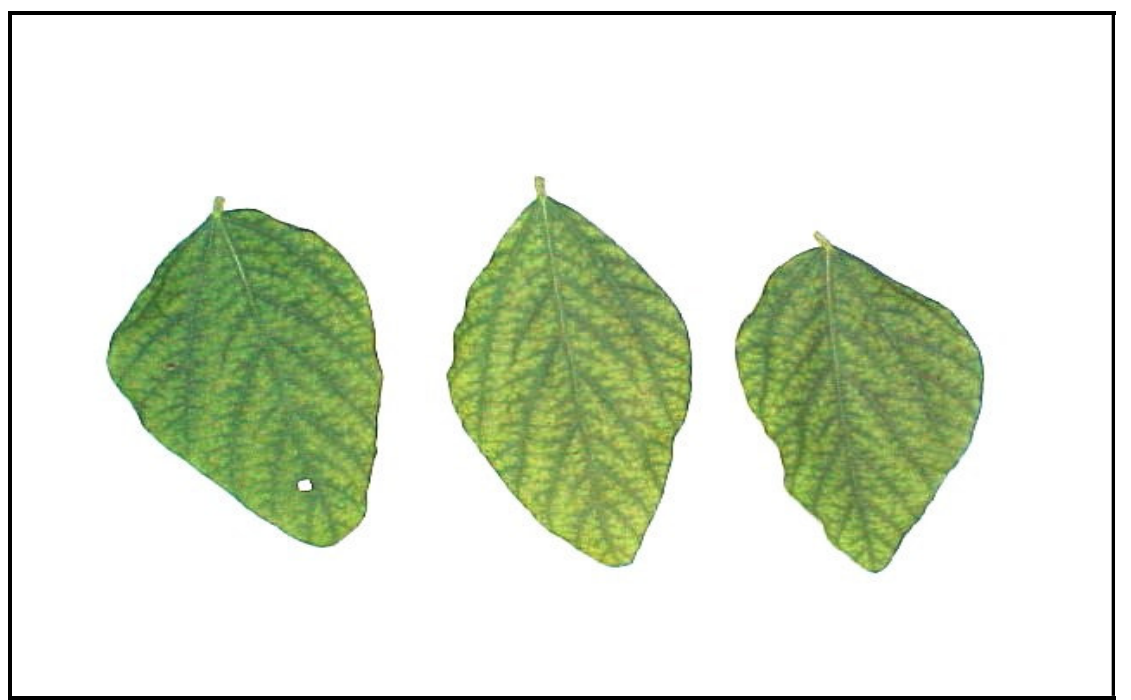

FIGURA 1 - Folhas de soja com sintomas de distúrbios nutricionais coletadas em lavoura da cultivar CD 202, no sul do Mato Grosso do Sul, Dourados, MS, 2000/2001.

\section{CONCLUSÕES}

1) Os índices DRIS calculados com base nos valores de referência desenvolvidos no trabalho e os compilados da literatura são diferentes, mostrando a necessidade de se desenvolver valores de referência regionais.
2) A concordância entre os diagnósticos feitos pelos métodos da diagnose visual e do DRIS, com base em sintomas foliares caracterizados como provocados por deficiência de Mn, indica a eficiência do método DRIS.

3) O DRIS é mais eficiente que o critério das faixas de concentração na interpretação de resultados analíticos de amostras de folhas.

\section{REFERÊNCIAS}

BATAGLIA, O.C.; DECHEN, A.R. Critérios alternativos para diagnose foliar. In: SIMPÓSIO AVANÇADO DE QUÍMICAE FERTILIDADE DO SOLO, 1., 1986, Piracicaba. Anais... Campinas: Fundação Cargill, 1986. p.115-136.

BATAGLIA, O.C.; DECHEN, A.R.; SANTOS, W.R. dos. Princípios da diagnose foliar. In: ALVAREZ V., V.H.; FONTES, L.E.F.F.; FONTES, M.P.F. (Ed.). O solo nos grandes domínios morfológicos do Brasil e o desenvolvimento sustentável. Viçosa: Sociedade Brasileira de Ciência do Solo/Universidade Federal de Viçosa, 1996. p.647-660.

BATAGLIA, O.C.; SANTOS, W.R. Efeito do procedimento de cálculo e da população de referência nos índices do Sistema Integrado de Diagnose e Recomendação (DRIS). Revista Brasileira de Ciência do Solo, Campinas, v.14, p.339-344, 1990.

BEAUFILS, E.R. Diagnosis and Recommendation Integrated System (DRIS); a general scheme for experimentation and calibration based on principles develop from research in plant nutrition. Pietermaritzburg: University of Natal, 1973. 132p. (Soil Science Bulletin, 1).

BORKERT, C.M.;YORINORI, J.T.; CORREA-FERREIRA, B.S.; ALMEIDA, A.M.R.; FERREIRA, L.P.; SFREDO, G.J. Seja o doutor da sua soja. Informações Agronômicas, Piracicaba, n.66, jun. 1994. Arquivo do Agrônomo, Piracicaba, n.5, p.1-16, jun. 1994.

COSTA, J.A.; MARQUEZAN, E. Características dos estádios de desenvolvimento da soja. Campinas: Fundação Cargill, 1982. 30p.

ELWALI, A.M.O.; GASCHO, G.J. Soil testing, foliar analysis and DRIS as guides for sugarcane fertilization. Agronomy Journal, Madison, v.76, p.466-470, 1984.

EMBRAPAAGROPECUÁRIA OESTE. Soja: recomendações técnicas para Mato Grosso do Sul e Mato Grosso. Dourados, 2000. 158p. (Embrapa Agropecuária Oeste. Circular Técnica, 6).

EMBRAPA SOLOS. Sistema brasileira de classificação de solos. Brasilia: Embrapa Produção da Informação, 1999. 412p.

KEOGH, J.L.; SABBE, W.B.; CAVINESS, C.E. Nutrient concentration of selected soybean cultivars. Communications in Soil Science and Plant Analysis, New York, v.3, p.29-36, 1972.

LEANDRO, W.M. Sistema Integrado de Diagnose e Recomendação (DRIS) para a cultura da soja (Glycine max L. Merril) na região de Rio Verde-GO. Goiânia: 1998. 157f. Tese (Doutorado em Agronomia, Produção Vegetal) - Universidade Federal de Goiás. MALAVOLTA, E.; VITTI, G.C.; OLIVEIRA, S.A. de. Avaliação do estado nutricional de plantas: princípios e aplicações. Piracicaba: Potafós, 1989. 201p.

PECK, T.R. Plant analysis for production agriculture. In: SOIL PLANT ANALYSIS WORKSHOP, 7., 1979, Bridgetown. Proceedings... Bridgetown, 1979. p.1-45. 
MAEDA, S.; RONZELLI Jr., P. Valores de referência do dris para...

SCHUTZ, C.J.; VILLIERS, J.M. Foliar diagnosis and fertilizer prescription in forestry - the DRIS system and its potential. South African Forestry Journal, Pretoria, v.141, p.6-12, 1987.

SUMNER, M.E. Interpretation of foliar analysis for diagnostic purposes. Agronomy Journal, Madison, v.41, p.343-348, 1979.

TISDALE, S.L.; NELSON, W.L.; BEATON, J.D.; HAVLIN, J.L. Soil fertility and fertilizers. 5.ed. New York: Macmillan, 1993. 634p. WALWORTH, J.L.; SUMNER, M.E. The Diagnosis and Recommendation Integrated System (DRIS). Advances in Soil Science, New York, v.6, p.149-88, 1987.

Recebido em 29/05/2003 Aceito em 05/04/2005 